\title{
Erster nationaler Grippeimpftag der Hausärzte am 29. Oktober 2004
}

\author{
U. Grüninger, Geschäftsführer Kollegium für Hausarztmedizin (KHM)
}

Die Organisationen der Schweizer Grundversorgerärzte führen am Freitag, 29. Oktober 2004, erstmals einen nationalen Grippeimpftag durch. An diesem Tag können sich alle interessierten Personen sowie all jene, die einer Risikogruppe (s. Artikel D. Müller) angehören, in den Praxen der teilnehmenden Hausärztinnen und Hausärzte ohne Voranmeldung gegen Grippe impfen lassen. Ziel der Aktion ist es, eine einfache Möglichkeit zur Grippeimpfung anzubieten, um eine Verbesserung der Impfquote zu erreichen.

Korrespondenz:

Dr. med. Ueli Grüninger

Landhausweg 26

CH-3007 Bern

E-Mail: ueli.grueninger@hin.ch

Internet: www.kollegium.ch
Die Grippeimpfung ist aufgrund von Studien wichtig für den Erhalt der Selbständigkeit und Lebensqualität von älteren Menschen und von Personen, die an chronischen Krankheiten leiden. Die Ärzteschaft nimmt bei der Förderung der Grippeimpfung eine zentrale Funktion ein. Allerdings stellte die Gesundheitsbefragung 2002 des Bundesamts für Statistik fest, dass nur eine Minderheit der Seniorinnen und Senioren unter 70 Jahren regelmässig zum Arzt geht. Dementsprechend ist die Impfquote bei der Risikogruppe der über 65jährigen mit weniger als $60 \%$ noch ungenügend (vgl. Artikel D. Müller). Um diese Präventionslücke $\mathrm{zu}$ schliessen und um eine einfache Möglichkeit für eine Grippeimpfung zu bieten, haben die Organisationen der Schweizer Hausärzte (das Kollegium für Hausarztmedizin KHM, die Schweizerischen Gesellschaften für Allgemeinmedizin SGAM, für Innere Medizin SGIM und für Pädiatrie SGP sowie die Vereinigung der praktischen Ärztinnen/Ärzte FMP) beschlossen, einen Nationalen Grippeimpftag einzuführen. FMH und BAG unterstützen diese Aktion aktiv.

Dieser nationale Grippeimpftag findet erstmals am Freitag, dem 29. Oktober 2004, statt. An diesem Tag können sich interessierte Personen in den Praxen der teilnehmenden Hausärztinnen und Hausärzte ohne Voranmeldung gegen Grippe impfen lassen. Für die an diesem Tag vorgenommene Grippeimpfung haben die Fachgesellschaften der Grundversorger ihren Mitgliedern einen Richtpreis von Fr. 25.- vorgeschlagen, alles inbegriffen. Für Personen, die einer Risikogruppe angehören, werden die Kosten für die Impfung von der Krankenkasse rückerstattet, sofern die Franchise bereits erreicht ist.

Die Schaffung eines landesweiten Angebots baut auf den Erfahrungen mit bisherigen regio- nalen Grippeimpfaktionen der Ärzteschaft auf. Der Nationale Grippeimpftag ist ausserdem ein geeignetes Instrument, um einerseits die Bevölkerung über die Grippe zu informieren, andererseits die Grippeimpfung als sinnvolle Gewohnheit bei den Zielgruppen der Grippepräventionskampagne zu etablieren. Sollte dieser Tag einen festen Platz im Präventionsangebot der Ärzteschaft bekommen, kann er auf kostengünstige Weise zu einer nachhaltigen Grippeprävention beitragen. Die Grundversorger, welche sich am Nationalen Grippeimpftag beteiligen, orientieren die Bevölkerung und ihre Kundinnen und Kunden via lokale Medien bzw. mittels Affichen in den Praxen, wo man sich ohne Voranmeldung gegen Grippe impfen lassen kann.

Ziel dieses bewusst niederschwelligen Angebotes ist es, die noch immer ungenügende Durchimpfquote in den für Grippe besonders anfälligen Risikogruppen zu verbessern. Das niederschwellige Angebot soll die noch nicht geimpften Personen in diesen Gruppen besonders ansprechen (z.B. jene, die nicht bereits regelmässig bei ihrem Hausarzt in Behandlung oder Kontrolle sind und deshalb nicht von den üblichen Grippeimpfprogrammen der Hausärzte bei deren Patienten erfasst werden).

Der Grippeimpftag der Hausärztinnen und Hausärzte wird dieses Jahr zum ersten Mal durchgeführt, und es gilt, Erfahrungen mit einer solchen landesweiten, aber regional organisierten Aktion zu sammeln. Dies insbesondere, weil die Nachfrage nicht im voraus bekannt sein wird. Letzteres wird von allen Beteiligten, den Impfinteressierten, aber auch von den Hausärztinnen und Hausärzten, welche sich auf freiwilliger Basis für die Bevölkerungsgesundheit engagieren, eine gewisse Flexibilität verlangen.

Ergänzende Auskünfte erhalten Sie

\section{a) über die Kontaktpersonen in den Fach- gesellschaften}

- SGAM: Kathrin Censier, Geschäftsführerin, Tel. 06148300 83, E-Mail: kathrin.censier@ sgam-ssmg.ch; 
- SGIM: Dr. med. Beat Rössler, Vorstand SGIM, Tel. 03263313 13, E-Mail: beat.roessler@ diac.ch;

- SGP: Marianne Neuenschwander, Generalsekretärin, Tel. 02635033 44, E-Mail: m.neuenschwander@swiss-paediatrics.org.

b) über die Geschäftsstelle des Kollegiums für Hausarztmedizin

Dr. med. Ueli Grüninger, Geschäftsführer, Tel. 03137006 71, E-Mail: ueli.grueninger@hin.ch.

\section{c) bei der Dokumentationsstelle} der Grippepräventionskampagne des BAG

E-Mail: grippe@bag.admin.ch, Fax 0313521471 (Anfragen, Bestellung des BAG-Infomaterials). Informationen rund um den Nationalen Grippeimpftag sowie die Grippepräventionskampagne des BAG sind ab 1. Oktober 2004 unter www. grippe.admin.ch bzw. www.influenza.admin.ch zu finden (siehe auch Webseite der Organisation «Unis contre la grippe», www.grippe.ch, als weitere Informationsquelle).

\title{
Nationale Grippepräventionskampagne - weitere Erfolge zeichnen sich ab
}

\author{
D. A. Müller
}

Korrespondenz:

Daniela A. Müller, dipl. biol. Bundesamt für Gesundheit Nationale Grippeprävention CH-3003 Bern

\section{Einleitung}

Influenza ist eine Infektionskrankheit, die jedes Jahr im Winter in Erscheinung tritt, sich epidemieartig über die ganze Schweiz ausbreitet und in der Bevölkerung zu erhöhter Morbidität und Mortalität führt. Im Gesundheitswesen führt die Grippe zu einer Zunahme der Arztkonsultationen und der Hospitalisierungen und stellt somit zusätzlich zu den soziomedizinischen eine ökonomische Belastung dar. In der Wirtschaft führen Grippeepidemien zu einer erhöhten Arbeitsplatzabwesenheit und zum Rückgang der Produktivität und verursachen somit indirekt höhere Kosten. Eine Schweizer Studie zum Kosten-Nutzen-Verhältnis von Präventionsmassnahmen gegen Grippe zeigt auf, dass die Grippeimpfung das effizienteste und kostengünstigste Mittel gegen Grippe darstellt und dass mit der Impfung bei Personen mit einem erhöhten Risiko infolge Grippe schwerwiegende Komplikationen und Todesfälle reduziert werden können [1]. Als Grundlage für eine nachhaltige Verminderung von Morbidität und Mortalität infolge Influenza ist eine Steigerung der Durchimpfung der Risikogruppen nötig. Die Bedeutung der Ziele der Nationalen Kampagne zur Förderung der Grippeprävention bei Personen mit einem erhöhten Komplikationsrisiko nach einer Influenzaerkrankung sowie beim Personal im Gesundheitswesen wird somit deutlich. Die vom
Bundesamt für Gesundheit im Jahr 2001 mit Unterstützung der FMH lancierte mehrjährige Kommunikations- und Informationskampagne steht vor dem Start ins vierte Jahr.

\section{Zunehmende Durchimpfung der Zielgruppen der Kampagne}

Personen über 65 Jahre lassen sich gemäss repräsentativer Telefonumfragen, die jährlich im Frühsommer durchgeführt werden, seit 1999 vermehrt gegen Grippe impfen. Während der Anteil der Geimpften im Herbst 1999 unter 50\% Prozent lag $(45,5 \% ; 95 \% \mathrm{CI} \pm 3,2 \%, \mathrm{n}=471)$, haben sich im Herbst 2003 deutlich mehr Personen $(57,8 \% ; 95 \% \mathrm{CI} \pm 3,0 \%, \mathrm{n}=1174)$ gegen Grippe impfen lassen. Erstmals überschritten wurde der 50\%-Anteil im Herbst 2001. Das Ziel der Nationalen Grippepräventionskampagne ist es, bei über 65jährigen eine Durchimpfung von $60 \% \mathrm{zu}$ erreichen. Inwiefern die Zunahme der Durchimpfung bei Seniorinnen und Senioren auf die Nationale Grippepräventionskampagne des BAG zurückzuführen ist, kann nicht abschliessend beurteilt werden. Sicher ist, dass die Grippeimpfung seit Lancierung der Kampagne im Jahr 2001 vermehrt thematisiert wird, sei es im Gesundheitswesen und von seiten der Berufsund Fachverbände, sei es in der Öffentlichkeit durch die Medien. 
Während die Grippeimpfung bei älteren Menschen im Verlauf der Jahre offensichtlich an Akzeptanz gewonnen hat, präsentiert sich die Situation beim Medizinal- und Pflegepersonal anders. Trotz positiver Erfahrungen mit der Impfung [2-4] und wissenschaftlicher Befunde, die zeigen, dass mit der Grippeimpfung Hospitalisierungen und Todesfälle bei Risikopersonen deutlich reduziert werden können [5-7], stösst die Impfung beim Medizinal- und Pflegepersonal auf Vorbehalte, und die Durchimpfung ist in diesen Berufsgruppen je nach Institution und je nach Sprachregion der Schweiz sehr unterschiedlich. Um ein genaueres Bild der Situation auf nationalem Niveau zu erhalten, wurden im Rahmen der Grippepräventionskampagne des BAG zwei Umfragen zur Grippeimpfung beim Medizinal- und Pflegepersonal durchgeführt. Die erste sogenannte Baselinestudie erfolgte im Frühling 2003 - und somit bevor das Medizinalund Pflegepersonal die primäre Zielgruppe der Grippepräventionskampagne 2003 war -, die zweite Umfrage erfolgte nach Abschluss der Kampagne 2003 im Frühling 2004. Die Studien wurden bei Mitgliedern des Schweizer Berufsverbands der Krankenschwestern und Krankenpfleger (SBK) und des Schweizer Berufs- und Fachverbands der Geriatrie-, Rehabilitationsund Langzeitpflege (SBGRL) durchgeführt.

Aufgrund der Umfragen hat sich im Herbst 2003 ein Viertel $(25,2 \%$; $95 \%$ CI $\pm 2,1 \%, \mathrm{n}=$ 1921) des berufstätigen Medizinal- und Pflegepersonals mit direktem Kontakt zu Patientinnen und Patienten gegen Grippe impfen lassen. Im Jahr vorher, im Herbst 2002, war die Durchimpfung tiefer $(21 \% ; 95 \% \mathrm{CI} \pm 1,6 \%, \mathrm{n}=2716)$. In der französischsprachigen Schweiz lag der Anteil der Geimpften im Herbst 2003 höher (30,1\%; $95 \%$ CI $\pm 3,7 \%$ ) als in der Deutschschweiz $(24,3 \%$; $95 \% \mathrm{CI} \pm 2,4 \%)$ und im Tessin $(26,6 \%$; $95 \%$ CI $\pm 4,4 \%$ ). Allerdings konnte in der Deutschschweiz zwischen Herbst 2002 und Herbst 2003 eine markante Steigerung festgestellt werden. Die Umfragen zeigen auf, dass die Durchimpfung mit zunehmendem Alter und mit zunehmender Dauer der Berufsausübung steigt. Am niedrigsten ist die Grippedurchimpfung bei Personen, die sich noch in Ausbildung befinden. Während die Grippedurchimpfung bei gleichen Institutionen, zum Beispiel bei Spitälern, grosse Unterschiede aufweist, zeigen sich auch deutliche Unterschiede je nach Art der Institution. Der Anteil der Geimpften ist am höchsten beim Personal von Alters- und Pflegeheimen (32,3\%; $95 \% \mathrm{CI} \pm 3,7 \%)$. In Spitälern lassen sich gesamtschweizerisch $21,2 \%$ (95\% CI $\pm 3,4 \%$ ) gegen
Grippe impfen, wobei der Anteil in der Deutschschweiz im Vergleich zur französischen Schweiz und zum Tessin am tiefsten ist. Bei der Spitex beträgt die Durchimpfung 21,7\% (95\% CI $\pm 5,7 \%$ ).

Trotz der Zunahme der Grippegeimpften beim Medizinal- und Pflegepersonal innerhalb eines Jahres ist die Durchimpfung dieser Zielgruppe noch immer ungenügend. Zur Verminderung der Übertragung von Influenzaviren auf Personen mit einem erhöhten Komplikationsrisiko sowie zur Reduktion von Morbidität und Todesfällen infolge Grippe ist eine höhere Durchimpfung des Medizinal- und Pflegepersonals massgebend. Es wird aufgrund der Studien klar, dass zur Förderung der Grippeprävention weiterhin Impfaktionen am Arbeitsplatz nötig sind und dass gezielte Information des Medizinal- und Pflegepersonals durch ihresgleichen die besten Ergebnisse erzielen kann.

\section{Dabeisein und Mitmachen - die Kampagne 2004}

Die diesjährige Kampagne steht im Zeichen der Lebensqualität im Alter und richtet sich unter dem Motto «Dabeisein und Mitmachen» an aktive Seniorinnen und Senioren. Die erneute Ausrichtung der Kampagne auf die Zielgruppe der über 65jährigen hat zum Ziel, die bisher erreichten Resultate zu festigen und noch bestehende Lücken in der Durchimpfung zu schliessen. Die Öffentlichkeit, insbesondere die Risikogruppen der über 65jährigen und der Personen mit chronischen Erkrankungen, aber auch das Medizinalund Pflegepersonal, wird dieses Jahr mittels Hängeplakaten in den öffentlichen Verkehrsmitteln der zwölf bevölkerungsreichsten Agglomerationen der Deutschschweiz und des Tessins auf die Grippeimpfung aufmerksam gemacht. In der Romandie wird ein von der interkantonalen Organisation «Unis contre la grippe» konzipierter TV-Spot ausgestrahlt. Neu wurde für Arztpraxen und für alle Institutionen, die Grippeimpfungen anbieten, eine Karte in Postkartengrösse erarbeitet, die, mit einem Stempel versehen, an die Kundschaft versandt werden kann. Die Karte erinnert, entsprechend dem Motto der diesjährigen Kampagne «Dabeisein und Mitmachen», an eine rechtzeitige Grippeimpfung und soll den Impfakteuren als Einladung dienen. Wie in den Vorjahren stellt das BAG allen Interessierten drei verschiedene zielgruppenspezifische Informationsbroschüren zur Verfügung. Es sind dies eine Fachbroschüre, eine Broschüre für Patientinnen und Patienten sowie eine Broschüre für das 
Medizinal- und Pflegepersonal. Diese können unentgeltlich auf deutsch, französisch oder italienisch bei der Dokumentationsstelle Grippeprävention, 3006 Bern, bezogen werden. Die Bestellung kann telefonisch (031 35214 60), per Fax (031 35214 71) oder per E-Mail (grippe@ bag.admin.ch) erfolgen. Zusätzliche Informationen zur Grippepräventionskampagne sind unter www.grippe.admin.ch zu finden. Ein weiterer Service zugunsten der Öffentlichkeit wird von der Impfinfoline erbracht, die im Auftrag des BAG vom Medizinischen Beratungszentrum Medgate angeboten wird. Fachpersonen geben unter der Nummer 0844448448 Auskunft zu den Themen Grippe und Grippeimpfung. Diese Dienstleistung ergänzt auf sinnvolle Weise das bestehende BAG-Informationsangebot.

\section{Der nationale Grippeimpftag der Hausärztinnen und Hausärzte}

Die Ärzteschaft sowie Apothekerinnen und Apotheker nehmen bei der Förderung der Grippeprävention bei älteren Personen und bei Personen mit einem erhöhten Risiko infolge Influenza eine zentrale Funktion ein. Das Kollegium für Hausarztmedizin, die Schweizerischen Gesellschaften für Allgemeinmedizin, für Innere Medizin, für Pädiatrie (SGAM, SGIM, SGP), die Vereinigung der praktischen Ärztinnen/Ärzte (FMP) und die FMH haben deshalb beschlossen, einen nationalen Grippeimpftag einzuführen, der erstmals am Freitag, dem 29. Oktober 2004, stattfinden wird. An diesem Tag können sich interessierte Personen für einen Richtpreis von Fr. 25.- in vielen Hausarztpraxen ohne Voranmeldung gegen Grippe impfen lassen. Der nationale Grippeimpftag stellt eine positive Ergänzung der Aktivitäten im Rahmen der Nationalen Grippepräventionskampagne dar. Das BAG begrüsst das Engagement der Grundversorgerorganisationen und unterstützt diese Aktion aktiv. Weitere Informationen zum nationalen Grippeimpftag finden Sie im Artikel von U. Grüninger vom Kollegium für Hausarztmedizin in dieser Ausgabe.

\section{Indikation für die Grippeimpfung}

Die Grippeimpfung wird empfohlen für Personen mit einem erhöhten Komplikationsrisiko nach einer Influenzaerkrankung und richtet sich an folgende Zielgruppen (kassenpflichtig) [8]:
- Personen über 65 Jahre;

- Personen (Kinder und Erwachsene) mit chronischen Herz- oder Lungenerkrankungen, chronischem Asthma, angeborener Fehlbildung des Herzens, zystischen Fibrosen, chronischen Stoffwechselstörungen (u.a. Diabetes), Niereninsuffizienz, Hämoglobinopathie oder Immunsuppression;

- Personen (Kinder und Erwachsene), die regelmässig medizinische Betreuung benötigen oder im Verlauf des Jahres hospitalisiert waren.

Die Grippeimpfung wird im weiteren auch bei folgenden Personen empfohlen (nicht kassenpflichtig unter 66 Jahren):

- Bewohner und Bewohnerinnen von Altersund Pflegeheimen und Patienten und Patientinnen in Einrichtungen für chronisch Kranke;

- Medizinal- und Pflegepersonal sowie alle Personen mit direktem Kontakt zu Patienten und Patientinnen und/oder Bewohnern und Bewohnerinnen von Spitälern, Kliniken oder Arztpraxen, bei der Hauspflege, in Alters- und Pflegeheimen sowie in Kurhäusern;

- Personen, die in nahem Kontakt stehen zu Risikopersonen oder mit ihnen im gleichen Haushalt leben (Kinder inbegriffen).

Eine Grippeimpfung kann auch in Betracht gezogen werden:

- bei allen Personen, die das Gripperisiko einschränken oder eine längere Abwesenheit am Arbeitsplatz verhindern möchten.

Schwangeren wird die Impfung bei Bestehen eines Grundleidens (chronische Herz-, Lungen-, Nieren- oder Stoffwechselerkrankungen) empfohlen. Die Impfung sollte nach Rücksprache mit der Ärztin/dem Arzt erst ab dem zweiten Schwangerschaftsdrittel erfolgen.

\section{Kontraindikation}

Bei einer Überempfindlichkeit auf Hühnereiweiss oder auf einen Inhaltsstoff der Impfung sollte auf die Grippeimpfung verzichtet werden. In diesen Fällen kann eine Prophylaxe oder bei Auftreten einer Grippeerkrankung eine Behandlung mit antiviralen Medikamenten in Betracht gezogen werden. Personen mit Fieber dürfen erst nach dem Abklingen der Symptome geimpft werden. Fieberfreie oder fast fieberfreie, leichte Erkrankungen stellen hingegen keine Kontraindikation dar. 


\section{Grippeimpfstoffe der Saison 2004/05}

Grippeimpfstoffe enthalten in der Regel zwei Influenza-A- und einen Influenza-B-Stamm. Der Impfstoff der Saison 2004/05 enthält folgende Komponenten:

- Antigene analog zu Influenza A/New Caledonia/20/99 (H1N1);

- Antigene analog zu Influenza A/Fujian/ 411/2002 (H3N2);

- Antigene analog zu Influenza B/Shanghai/ $361 / 2002$.

Die Zusammensetzung der Impfstoffe wird alljährlich durch eine Expertenkommission der WHO neu festgelegt. Als Grundlage dienen die Angaben $\mathrm{zu}$ den zirkulierenden Grippevirusstämmen aus der weltweiten Influenzaüberwachung. Für den auf der nördlichen Hemisphäre zum Einsatz gelangenden Impfstoff fällt der Entscheid bezüglich der Zusammensetzung jeweils im Februar. Je besser die zirkulierenden Influenzaviren mit den Impfstoffkomponenten übereinstimmen, desto wirksamer schützt die Grippeimpfung vor der Grippe.

\section{Art der Grippeimpfstoffe}

(Stand: September 2004)

In der Schweiz sind verschiedene parenteral zu applizierende Grippeimpfstoffe erhältlich:

- Splitimpfstoffe enthalten Influenzaviruspartikel in aufgebrochener Form (inklusive der beiden Oberflächenantigene Hämagglutinin und Neuraminidase): Fluarix ${ }^{\circledR}$, Mutagri $^{\circledR}{ }^{\circledR}$;

- Subunitimpfstoffe enthalten nur die beiden Oberflächenantigene Neuraminidase und Hämagglutinin: Influvac ${ }^{\circledR}$;

- virosomale Impfstoffe, bei denen die beiden Oberflächenantigene Neuraminidase und Hämagglutinin Teil einer Membran sind, die von den Immunzellen besser erkannt wird: Influvac plus ${ }^{\circledast}$, Inflexal $^{\circledR} V$.

\section{Produkte auf dem Schweizer Markt}

Momentan stehen auf dem Schweizer Markt fünf Influenzaimpfstoffe zur Verfügung, die aus Sicht des BAG alle gleichwertig sind: Fluarix ${ }^{\circledast}$ (GlaxoSmithKline AG), Influvac ${ }^{\circledR}$ (Solvay Pharma AG), Influvac $^{\circledR}$ plus (Solvay Pharma AG), Inflexal ${ }^{\circledR} \mathrm{V}$ (Berna Biotech AG), Mutagrip ${ }^{\circledast}$ (Pro Vaccine).

\section{Literatur}

1 Bundesamt für Gesundheit. Epidemiologie und Infektionskrankheiten. Ökonomische Analyse einer durchschnittlichen Grippeepidemie in der Schweiz. Bull BAG/OFSP 2003;46:818-22.

2 Govaert TM, Dinant GJ, Aretz K, Masurel N, Sprenger MJ Knottnerus JA. Adverse reactions to influenza vaccine in elderly people: randomised double blind placebo controlled trial. Br Med J 1993;307:988-90.

3 Margolis KL, Nichol KL, Poland GA, Pluhar RE. Frequency of adverse reactions to influenza vaccine in the elderly. A randomized, placebocontrolled trial. JAMA 1990;264:1139-41.

4 Nichol KL, Margolis KL, Lind A, Murdoch M, Mcfaden R, Hauge M. Side effects associated with influenza vaccination in healthy working adults. A randomized, placebo-controlled trial. Arch Intern Med 1996;156:1546-50.

5 Carman WF, Elder AG, Wallace LA, McAulay K, Walker A, Murray GD, Stott DJ. Effects on influenza vaccination of health-care workers on mortality of elderly people in long-term care: a randomised controlled trial. Lancet 2000; 355:93-7.

6 Christenson B, Lundbergh P, Hedlund J, Ortqvist A. Effects of a large-scale intervention with influenza and 23-valent pneumococcal vaccines in adults aged 65 years or older: a prospective study. Lancet 2001;357:1008-11.

7 Potter J, Stott DJ, Roberts MA, Elder AG, O'Donnell B, Knight PV, Carman WF. Influenza vaccination of health care workers in long-termcare hospitals reduces the mortality of elderly patients. J Infect Dis 1997;175:1-6.

8 Bundesamt für Gesundheit, Arbeitsgruppe Influenza, Schweizerische Kommission für Impffragen. Empfehlungen zur Grippeprävention. In: Bundesamt für Gesundheit (Hrsg.). Infektionskrankheiten. Diagnose und Bekämpfung. Suppl. XIII. Bern: Bundesamt für Gesundheit; 2000. 